\title{
The Hausdorff dimension of some snowflake-like recursive constructions
}

\author{
By S. D. Jacka and J. C. Croft \\ Statistics Department, University of Warwick, Coventry CV4 7AL
}

April 20, 2011

\begin{abstract}
Fractal subsets of $\mathbb{R}^{n}$ with highly regular structure are often constructed as a limit of a recursive procedure based on contractive maps.

The Hausdorff dimension of recursively constructed fractals is relatively easy to find when the contractive maps associated with each recursive step satisfy the Open Set Condition (OSC). We present a class of random recursive constructions which resemble snowflake structures and which break the OSC. We calculate the associated Hausdorff dimension and conjecture that an a.s. deterministic exact Hausdorff function does not exist.
\end{abstract}




\section{Introduction}

A recursive construction is defined as the limit of a sequence of sets, each of which is derived from its predecessor in the sequence by a simple step or rule. When the recursive step is deterministic, the resulting construction will often be self-similar, such as the Sierpinski gasket or carpet. The recursive step may, however, contain some randomisation. If the randomisation is conducted in a certain way, the resulting construction can be called statistically self-similar (see [12] for details).

Mandelbrot [16] pointed out the use of such sets as models of naturally occurring phenomena, such as snowflake crystals.

Restricting our attention to $\mathbb{R}^{n}$ from now on, in its simplest form the recursive step may be formulated in terms of a set of contractive maps $\left\{\phi_{i}\right\}_{i \in I}$. If we begin the recursion with a non-empty, compact set $K$ we can write each step in the construction as

$$
K_{j}=\bigcup_{\underline{i} \in\{1, \cdots, n\}^{j}} \phi_{\underline{i}}(K)
$$

where $\phi_{\left(i_{1}, i_{2}, \cdots, i_{j}\right)}=\phi_{i_{j}} \circ \phi_{i_{j-1}} \circ \cdots \circ \phi_{i_{1}}$. Hence the limiting set can be written as

$$
K=\bigcap_{j=1}^{\infty} K_{j}
$$

The OSC is of great use when calculating the Hausdorff dimension of the limiting set, see [7] for examples. 
The Hausdorff dimension of deterministic OSC recursive constructions was calculated by Hutchinson [14] and a good description of the exact Hausdorff functions was obtained by, for example, Graf in [12].

In Mauldin and Williams [18] (also in [13]) the recursive step is randomised in a heterogeneous way. That is a random (according to a law $\mu$ say) i.i.d. selection of contractive maps (satisfying the OSC) is applied to each scaled copy of the original set in every recursive step. Here it is possible to ensure the a.s. existence not only of the Hausdorff dimension $\alpha$ but also of a (deterministic) exact Hausdorff function $h$ for the resulting set $F$ where $\alpha$ and $h$ satisfy

$$
\int \sum_{i} \operatorname{Lip}\left(\phi_{i}\right)^{\alpha} d \mu=1
$$

and

$$
h(t)=t^{\alpha}(\log |\log (t)|)^{\theta}
$$

with $\theta \leq 1-\frac{\alpha}{n}<1$.

In Sec. 4 we introduce a collection of homogeneous random recursive models for snowflake crystals which breaks the OSC. Homogeneous refers to the application of the same random selection of contractive maps to each copy of the original set at each recursive step. Recursive constructions of a related homogeneous type were studied by Bedford in [3].

No general theory exists for finding the Hausdorff dimension of non-OSC constructions. We use special properties of the collection to find a random 
exact Hausdorff function. We can then use this function to calculate the Hausdorff dimension.

In Sec. 5 a condition is given (the rotation or R-condition) which splits the class into those models for which we can find the Hausdorff dimension explicitly and those for which we cannot. Under the condition, the Hausdorff dimension is found to be

$$
\mathbb{E}\left(B_{1}\right)+1
$$

where $B_{i}$ is a random variable associated with the number of contractive maps in the selection for stage $i$.

We then use a triangle covering approach to refine the proof of the above result to apply to those models which break the condition. The Hausdorff dimension of these models is given by the largest characteristic exponent of a random matrix product.

In sec. 7 we comment that the given results are in some sense strong, that is they do not depend on any nice properties of the random sequence of recursive steps (independence, stationarity, etc) but merely on the sequence itself. We also comment on other similar models which break the OSC and conjecture that no similar results are possible. 


\section{Hausdorff dimension and exact Hausdorff func- tion}

Let $U \subseteq \mathbb{R}^{n}$. If $\left\{U_{i}\right\}_{i \geq 1}$ is a countable (or finite) collection of sets then we say that $\left\{U_{i}\right\}_{i \geq 1}$ is a $\delta$-cover of $U$ if

$$
U \subset \bigcup_{i} U_{i} \text { and }\left|U_{i}\right| \leq \delta \text { for all } i \geq 1
$$

where $\left|U_{i}\right|=\operatorname{diam}\left(U_{i}\right)$ using the usual metric on $\mathbb{R}^{n}$. Let $s>0$ and define

$$
H_{\delta}^{s}(U)=\inf \left\{\sum_{i}\left|U_{i}\right|^{s}:\left\{U_{i}\right\} \text { is a } \delta \text {-cover of } F\right\} \text {. }
$$

Now set

$$
H^{s}(U)=\lim _{\delta \rightarrow 0} H_{\delta}^{s}(U)
$$

It is easily shown that the limit exists and also that $H^{s}(\cdot)$ is indeed a measure (Hausdorff $s$-measure).

For a set $U \subset \mathbb{R}^{n}$ define the Hausdorff Dimension to be the unique number $\operatorname{dim}_{H}(U)$ such that

$$
H^{s}(U)=\left\{\begin{array}{cc}
\infty & s<\operatorname{dim}_{H}(U) \\
0 & s>\operatorname{dim}_{H}(U)
\end{array}\right.
$$

The definition of Hausdorff measure above can be generalized to include any positive increasing function $f:[0, \infty) \rightarrow[0, \infty)$, (rather than just powers) of the diameters of the covering sets with the property that $f(x) \rightarrow 0$ as $x \rightarrow 0$ 


$$
H_{\delta}^{f}(U)=\inf \left\{\sum_{i} f\left(\left|U_{i}\right|\right):\left\{U_{i}\right\} \text { is a } \delta \text {-cover of } U\right\}
$$

The analogue of the dimension here is an exact Hausdorff function, a function $h$ such that

$$
0<H^{h}(U)<\infty
$$

The theory of these measures (which are in general non $\sigma$-finite) has proceeded largely due to the work of A. S. Besicovitch and his students. A fine technical work on the theory of Hausdorff measures is Rogers [23].

The contractive maps most commonly used in the formulation of recursive constructions are called similitudes, essentially just scaled isometries.

(Similitude) A function $S: X \rightarrow X$ on a metric space $(X, d)$ is called a similitude if $d(S(x), S(y))=r d(x, y)$ for all $x, y \in X$ and for some fixed $r$.

\section{Random Recursions}

In this sec. we will outline the formulation of a homogeneous random recursion.

Let $\mathcal{S}=\left(S_{i}\right)_{i=1}^{N}$ be a set of similitudes on $\mathbb{R}^{M}$, let $\mu$ be a probability measure on the power set of $\{1, \ldots, N\}$ and let $K$ be a non-empty, compact subset of $\mathbb{R}^{M}$ with a non-empty interior. We specify the construction as follows; $K_{0}=K$, now we choose an subset $T_{1} \subseteq\{1, \ldots, N\}$ according to $\mu$ and set 


$$
K_{1}=\bigcup_{j \in T_{1}} S_{j}\left(K_{0}\right)
$$

Now choose another set $T_{2} \subseteq\{1, \ldots, N\}$ according to $\mu$ and independently of $T_{1}$, we can now write

$$
K_{2}=\bigcup_{l \in T_{2}} \bigcup_{j \in T_{1}} S_{l}\left(S_{j}\left(K_{0}\right)\right)
$$

Continuing the recursion yields a sequence of sets $\left(K_{n}\right)_{n \geq 1}$ and we may define our recursive construction to be $F$ where

$$
F=\lim \sup K_{n}=\bigcap_{n \geq 1} \bigcup_{j \geq n} K_{j}
$$

\section{A class of random recursive constructions}

Let $H$ be a regular hexagon of radius 1 , let $D$ be its 'diameter' and $v_{1}, \ldots, v_{6}$ its vertices as shown in Fig 1 . Define the similitudes $\phi_{1}, \ldots, \phi_{6}$ by requiring that $\phi_{i}$ maps $H$ to $H_{i}$ as shown in Fig 1.

\section{Figure 1 here}

We will call a random recursive construction procedure a $G H$ (general hexagonal) procedure if (in the notation of the previous Sec.);

1. $K$ is the unit hexagon $H$ centred at the origin

2. the set of similitudes is $\left\{\phi_{1}, \ldots, \phi_{6}\right\}$ : 
3. the selection law satisfies $\mu\left(\{1,2\} \subseteq T_{i}\right)=1$.

\section{Figure 2 here}

An example of a GH construction appears in Fig. 2. The associated selection law $\mu$ is given by

$$
\mu(\{1,2\})=1-\mu(\{1,2,3,6\})=p
$$

for $0<p<1$. We refer to this particular model as SH (special hexagonal). Despite the simplicity of this model, simulations produce snowflake-like figures of great variety and complexity. Some examples are shown in Fig. 3.

Figure 3 here

In fact, simulations from any model in the class GH, even one with asymmetric replacements, appear to produce snowflake-like figures. Fig. 4 contains simulations from different models within the GH class.

\section{Figure 4 here}

Let $F(H)$ be the limiting set obtained using a particular GH procedure as given above and let $F(D)$ be the closure of the limiting set obtained using the identical procedure applied to an initial set $K=D$, the diameter of the hexagon $H$.

\section{Lemma 4.1 (Equivalence lemma)}

$$
F(H)=F(D)
$$




\section{Proof}

Clearly $D \subset H$ and $F(H)$ is closed hence

$$
F(D) \subseteq F(H)
$$

Conversely if $x \in F(H)$ take $i_{1}, \cdots, i_{n}$ such that $i_{j} \in T_{j}$ for $1 \leq j \leq n$ and $x \in \phi_{i_{n}} \circ \phi_{i_{n}-1} \cdots \phi_{i_{1}}(H)$ Now $d\left(x, \phi_{i_{n}} \circ \phi_{i_{n}-1} \cdots \phi_{i_{1}}(0)\right) \leq 2^{-n}$ and the origin $0 \in D$. Since $x$ is arbitrary in $F(H)$ and $F(D)$ is closed then $x \in F(D)$ and hence $F(D)=F(H)$.

For convenience we will use the initial set $D$ in all subsequent constructions.

\section{$5 \quad$ A covering using triangles}

The most important consideration in calculating the Hausdorff dimension of a recursive construction is keeping track of the number of scaled copies of the original set $(D)$ that are present in any particular stage. This is simple when the OSC holds as no elements in any stage overlap. In a general GH model there is non-trivial overlapping of the form shown in Fig. 5 for the SH model.

Figure 5 here

However, with this particular model the largest number of copies of $D$ (lines) which may overlap (coincide) is 2 . In fact there is a condition which identifies this property in GH models. 
A GH construction is said to satisfy the $R$-condition if no replacement used in the construction overlaps with its image under a rotation of $\pi$ about its centre, except at the line being replaced.

Under the R-condition the maximum overlap (coincidence) of elements (lines) is 2 , without the R-condition the maximum overlap is unbounded.

We will now introduce a method to keep track of the total number of elements (lines) present at any particular stage based on covering with triangles.

We will use triangles of side length $2^{-n}$ to cover $D_{n}$ (the $n$th stage in the recursive construction with initial set $D$ ), in which the elements are themselves of length $2^{-n}$. Note the following facts

1. elements of $D_{n}$ will fill the edges of each covering triangle

2. all future branching from the elements in a triangle will clearly not be contained within the triangle, but

3. the future branching within a particular triangle is dependent on the composition of the edges of that triangle and no other

The possible arrangements of elements of $D_{n}$ on the edges of an appropriately sized triangle (up to rotation and reflection) are shown in Fig. 6.

\section{Figure 6 here}

Note that at each stage each covering triangle generates at most 4 covering triangles of the next stage. The types of the new triangles depend only on 
the previous triangle type and the form of the replacement. Note that we ignore any 'empty' triangles that may be generated.

Also, in any particular GH procedure certain configurations of line segments may never occur. For instance in the $\mathrm{SH}$ example the triangles numbered 20 through to 23 are never needed to cover a part of any structure which may occur. In such a case, to simplify the notation, we assume the list of triangle types and the 'transition' matrices below are trimmed so that redundent types are omitted.

We may now analyse each $D_{n}$, calculating for example the number of elements or the number of directed elements (counting 2 or more for an overlapped element), just by counting the numbers of each type of covering triangle at each stage. These are dependent only on the initial distribution of triangle types ${ }^{1}$ and the sequence of choices of contractive maps (be it deterministic or random).

\section{Figure 7 here}

If we let $t_{n}$ be the column vector of counts of each (non-redundant) triangletype needed to cover $D_{n}$ in the method described above then

$$
t_{n}=M_{n} t_{0}
$$

where $M_{n}=P^{T_{n}} P^{T_{n-1}} \cdots P^{T_{1}}$ and $P_{k, l}^{T_{i}}$ is the number of triangles of type $k$ generated from a single triangle of type $l$ by the application of the similitudes

\footnotetext{
${ }^{1}$ For a $\mathrm{GH}$ procedure the initial distribution is given by the vector $(1,1,0, \cdots, 0)$
} 
indexed by $T_{i}$.

Using this cover, the number of triangles required at stage $n$ is $1^{t} M_{n} t_{0}$.

For example in the SH model, the number of lines, counting each overlapped line as two, grows exactly as $2^{n+X_{n}}$ where $X_{n}$ is the number of times the 4-line replacement is used up to stage $n$. If we let $\pi$ be the fixed vector listing the number of edges in each triangle type (counting multiplicity),

$$
\pi^{t}=(1,1,2,1,2,2,2,3,3,3,3,4,4,5,5,3,3,6,4,3,3,4,4)
$$

it is clear that $\pi^{t} M_{n} t_{0}=2^{n+X_{n}} \pi^{t} t_{0}$ and that

$$
\frac{\pi^{t} M_{n} t_{0}}{6} \leq 1^{t} M_{n} t_{0} \leq \pi^{t} M_{n} t_{0}
$$

Hence the number of triangles of diameter $2^{-n}$ required to cover $D_{n}$, and hence the number of lines contained in $D_{n}$, grows as $2^{(2-p) n+\mathrm{O}(n)}$. It follows immediately that $(2-p)$ is an upper bound for the Hausdorff dimension any realisation of the $\mathrm{SH}$ model.

Figure 8 here

\section{Exact Hausdorff function for $\mathrm{SH}$}

Theorem 6.1 If $F$ is a recursive construction formed via the SH model and we denote by $X_{n}$ the number of times the set $\{1,2,3,6\}$ is chosen up to stage $n$ then the function $\phi$, given by

$$
\phi\left(2^{-n}\right)=2^{-\left(n+X_{n}\right)}
$$


$\left(\phi(x)\right.$ being obtained by interpolation whenever $\left.2^{-n}<x<2^{-n+1}\right)$, is an exact Hausdorff function.

\section{Proof}

\section{Lower bound}

Consider a set $U \in \mathbb{R}^{2}$ of diameter $d$ with

$$
2^{-(n+1)}<d \leq 2^{-n}
$$

By considering all the hexagons of diameter $2^{-(n+1)}$ which can intersect $U$, we can see (Fig. 8) that the most line segments of length $2^{-(n+2)}$ in $D_{n+2}$ which can be killed (i.e. they are covered and all their 'decendents' are covered) by $U$ is 154 .

Now suppose that $\left(U_{i}\right)_{i \in I}$ is a $\delta$-cover of $F$ with $2^{-(N+1)}<\delta \leq 2^{-N}$ and let

$$
n_{0}=\min \left\{n: \exists i \in I \text { with } 2^{-(n+1)}<\operatorname{diam}\left(U_{i}\right) \leq 2^{-n}\right\} .
$$

Now define $n_{i}$ recursively by

$$
n_{i+1}=\min \left\{n>n_{i}: \exists i \in I \text { with } 2^{-(n+1)}<\operatorname{diam}\left(U_{i}\right) \leq 2^{-n}\right\} .
$$

Let $\pi_{0}$ be the proportion of line segments of length $2^{-\left(n_{0}+2\right)}$ in $D_{\left(n_{0}+2\right)}$ killed by sets in the cover with diameters in the range $\left(2^{-\left(n_{0}+1\right)}, 2^{-n_{0}}\right]$, then it follows that the number of such sets in the cover is at least

$$
\frac{2^{\left(n_{0}+2\right)+X_{\left(n_{0}+2\right)}} \pi_{0}}{154}
$$


Define $\pi_{1}$ as the proportion of those line segments of length $2^{-\left(n_{1}+2\right)}$ in $D_{\left(n_{1}+2\right)}$ which have not already been killed by covering sets of diameter $>2^{-n_{1}}$ but which are killed by sets of diameter in the range $\left(2^{-\left(n_{1}+1\right)}, 2^{-n_{1}}\right]$ The remaining number of lines not covered at stage $n_{1}$ is

$$
\left(1-\pi_{0}\right) 2^{\left(n_{0}+2\right)+X_{n_{0}+2}} 2^{\left(n_{1}-n_{0}\right)+\left(X_{n_{1}+2}-X_{n_{0}+2}\right)} .
$$

Using the same arguments, the number of covering sets in the range $\left(2^{-\left(n_{1}+1\right)}, 2^{-n_{1}}\right]$ is at least

$$
\frac{2^{\left(n_{1}+2\right)+X_{n_{1}+2}} \tilde{\pi_{1}}}{154}
$$

where $\tilde{\pi_{1}}=\left(1-\pi_{0}\right) \pi_{1}$ and $0 \leq \pi_{1} \leq 1$. Continuing for $n_{2}<n_{3}<\cdots$ we obtain

$$
\begin{aligned}
H_{\phi}^{\delta}(F) \geq & \liminf _{N \rightarrow \infty} \inf _{\tilde{\pi}}\left(\left(\frac{2^{\left(n_{0}+2\right)+X_{n_{0}+2}}}{154} \tilde{\pi_{0}} \phi\left(2^{-\left(n_{0}+1\right)}\right)\right)+\right. \\
& \left.\left(\frac{2^{\left(n_{1}+2\right)+X_{n_{1}+2}}}{154} \tilde{\pi}_{1} \phi\left(2^{-\left(n_{1}+1\right)}\right)\right)+\cdots\right)
\end{aligned}
$$

where the infimum is taken over $\tilde{\pi}=\left(\tilde{\pi_{0}}, \tilde{\pi_{1}}, \tilde{\pi_{2}}, \cdots\right)$ such that $\Sigma_{i} \tilde{\pi}_{i}=1$ and each $\tilde{\pi}_{j} \geq 0$.

Clearly, for any $\epsilon>0$ there will be a $k \in \mathbb{N}$ such that

$$
H_{\phi}^{\delta}(F) \geq \frac{2^{\left(n_{k}+2\right)+X_{n_{k}+2}}}{154} \phi\left(2^{-\left(n_{k}+1\right)}\right)-\epsilon
$$

Since $n_{k}$ is bounded below by $N$ which tends to $\infty$ as $\delta \rightarrow 0$,

$$
H_{\phi}(F) \geq \liminf _{n \rightarrow \infty} 2^{n+X_{n}} \phi\left(2^{-n}\right) / 154
$$




\section{Upper bound}

By considering the simple coverings of $F$ given by placing a regular hexagon of diameter $2^{-n}$ over each line segment at stage $n$, it is clear that

$$
H_{\phi}(F) \leq \liminf _{n \rightarrow \infty} 2^{n+X_{n}} \phi\left(2^{-n}\right)
$$

Hence to identify an exact Hausdorff function $\phi$ it is enough to ensure that

$$
0<\liminf _{n \rightarrow \infty} 2^{n+X_{n}} \phi\left(2^{-n}\right)<\infty
$$

Now notice that this may be guaranteed by setting

$$
\phi\left(2^{-n}\right)=2^{-\left(n+X_{n}\right)}
$$

and interpolating for $\phi(x)$ when $2^{-n}<x<2^{-n+1}$. This completes the proof of Theorem 6.1.

We can now obtain $\operatorname{dim}_{H}(F)$ via the exact Hausdorff function $\phi$ in Theorem 6.1, using the facts that,

$$
\frac{x^{r}}{\phi(x)} \rightarrow \infty \quad \text { as } \quad x \rightarrow 0 \Rightarrow \operatorname{dim}_{H}(F) \geq r
$$

and

$$
\frac{x^{r}}{\phi(x)} \rightarrow 0 \quad \text { as } \quad x \rightarrow 0 \Rightarrow \operatorname{dim}_{H}(F) \leq r
$$

\section{Corollary 6.2}

$$
\operatorname{dim}_{H}(F)=2-p
$$




\section{Proof}

Let $r<2-p$. Using the above remark we simply require that

$$
\frac{\left(2^{-n}\right)^{r}}{\phi\left(2^{-n}\right)} \rightarrow \infty
$$

but

$$
\frac{\left(2^{-n}\right)^{r}}{\phi\left(2^{-n}\right)}=2^{-\left(n(r-1)-X_{n}\right)}
$$

and the Strong Law of Large Numbers states that $\frac{X_{n}}{n} \rightarrow 1-p$ with probability 1 . Hence

$$
2^{-\left(n(r-1)-X_{n}\right)} \sim 2^{-n(r-1-1+p)}=2^{n(r-(2-p))} \rightarrow \infty
$$

as $n \rightarrow \infty$. Hence $r$ is a lower bound for $\operatorname{dim}_{H}$ for any $r<2-p$. The reverse follows similarly.

\section{Hausdorff Dimension for GH}

Theorem 6.3 If $F$ is a set constructed by a GH scheme satisfying the $R$ condition then an exact Hausdorff function, $h$, is given by

$$
h\left(2^{-n}\right)=R_{n}^{-1}
$$

and $h(t)$ is calculated by linear interpolation for $2^{-n} \geq t>2^{-(n+1)}$ where $R_{n}$ is the number of distinct lines at stage $n$ of the construction. 


\section{Proof}

We simply mimic the proof of Theorem 6.1.

Theorem 6.4 If $F$ is a set constructed by a GH scheme satisfying the $R$ condition

$$
d_{H}(F)=E(B)+1
$$

where $B$ is a random variable whose distribution is given by

$$
B=\left\{\begin{array}{lll}
0 & \text { w.p. } & p_{2} \\
\log (3) / \log (2)-1 & \text { w.p. } & p_{3} \\
1 & \text { w.p. } & p_{4}
\end{array}\right.
$$

and $p_{2}, p_{3}, p_{4}$ are the probabilities of choosing replacements with 2,3 or 4 lines respectively.

\section{Proof}

Notice that we can write

$$
R_{n}=2^{n+\sum_{i=1}^{n} B_{i}}
$$

where the $B_{i}$ are i.i.d. random variables each distributed as $B$. The result now follows in a similar manner to corollary 6.2 .

\subsection{Outside the R-Condition}

When a GH construction breaks the R-condition, it is easy to see that the argument for the exact Hausdorff function is still valid with the number of 
lines at stage $n,\left(2^{n+X_{n}}\right)$, replaced by $R_{n}$ (just defined to be the random number of distinct line segments, overlapped or not, at each stage $n$ ).

Using the triangle covering we can express $R_{n}$ simply as

$$
\pi M_{n} x
$$

where $M_{n}$ is a random product of the branching matrices associated with each replacement. To assure the existence of a power law governing the limiting behaviour of this product we will employ a version of Oseledec's theorem (first seen in [22]) as given in an excellent review of the topic in $[15]$.

Theorem 6.5 (Theorem 3.1 of [15]) Consider a random stationary sequence $\left\{A_{n}: n \geq 0\right\}$ of $d \times d$ real-valued matrices (on a probability space $(\Omega, \mathcal{F}, \mathbb{P}))$ and form the product

$$
A^{(n)}=A_{n-1} \cdots A_{0}
$$

There exist positive constants $\mu_{1}>\mu_{2}>\cdots>\mu_{r}$ and, for each $\omega \in \Omega$, an ordered decomposition of $\mathbb{R}^{d}$ into subspaces of strictly decreasing dimension

$$
\mathbb{R}^{d}=V_{\omega}^{1} \supset V_{\omega}^{2} \supset \cdots V_{\omega}^{r}=\{0\}
$$

such that for $1 \leq j \leq r-1$ and $v \in V_{\omega}^{j} \backslash V_{\omega}^{j+1}$ we have

$$
\lim _{n \rightarrow \infty} \frac{1}{n} \log \left\|A_{\omega}^{(n)} v\right\|=\mu_{j}
$$


Theorem 6.6 If $F$ is formed using a GH procedure then $\operatorname{dim}_{H}(F)$ is almost surely constant and is given by $\log _{2} \mu_{1}$ where $\mu_{1}$ is defined as in the previous theorem.

\section{Proof}

Using the identities from Sec. 5 and Theorem 6.5 it is clear that $R_{n}$ grows as $2^{n \log _{2} \mu_{1}+o(n)}$ and so again mimicking the proofs of 6.1 and 6.2 , it is clear the Hausdorff dimension can be derived from one of the exponents $\mu_{i}$ of Theorem 6.5. To show the correct exponent is $\mu_{1}$ we must show that the starting vector of triangle types $t_{0}(=(1,1,0, \cdots, 0)$ for $\mathrm{GH}$ constructions as given in Sec. 4) is in $V_{\omega}^{1} \backslash V_{\omega}^{2}$.

As the decomposition of $\mathbb{R}^{d}$ in theorem 6.5 is into subspaces there must exist $i \in \mathbb{N}$ such that the vector corresponding to one triangle type $i, b_{i}=$ $(0, \cdots, 0,1,0, \cdots, 0) \in V_{\omega}^{1} \backslash V_{\omega}^{2}$.

However, each triangle type can be considered as the superposition of up to 3 rotated copies of a type 1 and 3 rotated copies of type 2 , hence

$$
6 \pi^{t} M_{n} t_{0} \geq \pi^{t} M_{n} b_{i}
$$

for any $i$ and so $t_{0} \in V_{\omega}^{1} \backslash V_{\omega}^{2}$.

We can generalise the argument further since the exact Hausdorff function is given by $h\left(2^{-n}\right)=R_{n}^{-1}$ regardless of the scheme employed for choosing the form of the recursive step. 
In particular we can calculate the Hausdorff dimension of any GH model (explicitly under the R-condition) with a deterministic recursive step or a deterministically repeating sequence of recursive steps.

\section{$7 \quad$ Further comments}

The models in GH are based on the radii of a regular hexagon. It is natural to ask whether it is possible to extend the earlier results to models based on the regular $2 n$-agon for $n>3$.

A simple argument show that this is not possible for constructions based on $4 n$-agons with $n \geq 2$. In these cases no fixed upper bound exists for the number of stage $n$ elements a set of diameter close to $2^{-n}$ can cover. We conjecture that the same problem persists for any construction based on a $2 n$-agon with $n>3$.

It is thought that because of the simple form of the matrix product $M_{n}$ used to calculate $R_{n}$ in Theorem 6.6 , we may be able to evaluate (or closely bound) the exponent $\mu_{1}$.

We also conjecture that no a.s. deterministic exact Hausdorff function exists due to the homogeneous randomisation in GH models. This method of randomisation does not produce enough 'averaging' in the long run to be able to apply the same arguments as those in [13]. 


\section{Acknowledgments}

The authors wish to thank Ben Hambly for some useful discussions. The work was funded by an EPSRC research studentship.

\section{References}

[1] M. T. Barlow, B. M. Hambly (1996) Transition density estimates on scale irregular Sierpinski gaskets. Annals of the Institute of H. Poincaré 33 531-557

[2] M. Barnsley (1988) Fractals Everywhere Academic Press Inc., San Diego.

[3] T. Bedford (1989) On Weierstrass-like functions and random recurrent sets. Math. Proc. Cambridge Philos. Soc. 106 325-342.

[4] W. A. Bently, W. J. Humphries (1962) Snow Crystals. New York: Dover.

[5] J. C. Croft (1998) Line models for snowflake growth and their Hausdorff dimension. Proceedings of the 7th Vilnius Probability Meeting, TEV, Vilnius.

[6] J. C. Croft (1998) Some generalisations of nested fractals and associated diffusions. PhD. Thesis. University of Warwick 1999. 
[7] K. J. Falconer (1990) Fractal Geometry: Mathematical Foundations and Applications. Wiley, Chichester.

[8] K. J. Falconer (1987) Cut-set sums and tree processes. Proceedings of the American Mathematical Society 101 235-246

[9] K. J. Falconer (1988) The Hausdorff dimension of self-affine fractals. Mathematical Proceedings of the Cambridge Philosophical Society 103 $339-350$

[10] F. Family, D. E. Platt, T. Vicsek (1987) Deterministic growth model of pattern formation in dendritic solidification. Journal of Physics Letters 20 L1177-L1184

[11] P. Garik, R. Richter, J. Hautman, P. Ramanial (1985) Deterministic solutions of fractal growth. Physics Review A 32 3156-3159

[12] S. Graf (1987) Statistically self-similar fractals. Probability Theory and Related Fields 74 357-392

[13] S. Graf, R. D. Mauldin, S. C. Williams (1988) The exact Hausdorff dimension in random recursive constructions. Memoirs of the American Mathematical Society 381

[14] J. E. Hutchinson (1981) Fractals and self similarity. Indiana University Mathematics Journal 30 713-747 
[15] F. Ledrappier (1982) Quelques properties des exponents characteristiques. Springer Lecture Notes 1097

[16] B. B. Mandelbrot (1982) The fractal geometry of nature. Freeman, San Francisco.

[17] B. B. Mandelbrot (1992) Plane DLA is not self-similar; is it a fractal that becomes increasingly compact as it grows?. Physica A 191 95-107

[18] R. D. Mauldin, S. C. Williams (1986) Random recursive constructions: asymptotic geometric and topological properties. Transactions of the American Mathematical Society 295 325-346

[19] P. A. P. Moran (1946) Additive functions of intervals and Hausdorff measure. Mathematical Proceedings of the Cambridge Philosophical Society 42 15-23

[20] U. Nakaya - U.S. Army (1956) Properties of Single Crystals of Ice, Revealed by Internal Melting. U.S. Army Snow Ice and Permafrost Research Establishment, Research Paper 13.

[21] J. Nittmann, H. E. Stanley (1987) Non-deterministic approach to anistropic growth patterns with continuously tunable morphology: the fractal properties of some real snowflakes. Journal of Physics Letters 20 L1185-L1191 
[22] V. I. Oseledec (1968) A multiplicative ergodic theorem, Lyapunov characteristic number for dynamical systems. Transactions of the Moscow Mathematical Society 19 197-231

[23] C. A. Rodgers (1970) Hausdorff Measures. Cambridge University Press.

[24] A. Schief (1994) Separation properties for self-similar sets. Proceedings of the American Mathematical Society 122 111-115

[25] E. Seneta (1981) Non-negative Matrices and Markov Chains. Springer Verlag, New York Heidelberg Berlin.

[26] S. J. Taylor (1986) The measure theory of random fractals. Mathematical Proceedings of the Cambridge Philosophical Society 100 383-406 